\title{
X-RAY SPECTROSCOPY OF THE ULTRA-SOFT TRANSIENT 4U1543-47
}

\author{
H. van der Woerd ${ }^{1}$, N.E. White ${ }^{1}$, and S.M. Kahn ${ }^{2}$
}

1 EXOSAT Observatory, Astrophysics Division, Space Science Department of ESA, ESTEC, Postbus 299, 2200 AG Noordwijk, The Netherlands.

${ }^{2}$ Department of Physics, University of California, Berkeley, CA 94720, U.S.A.

\begin{abstract}
The X-ray transient $4 \mathrm{U} 1543-47$ was observed in 1983 by the EXOSAT observatory near the maximum of an outburst. The X-ray spectrum was measured using a gas scintillation proportional counter (GSPC) and a transmission grating spectrometer (TGS). Two emission line features are resolved. A broad (FWHM $2.7 \mathrm{keV}$ ) line at 5.9 $\mathrm{keV}$ is detected in the GSPC, which we interprete as a redshifted and broadened iron $\mathrm{K} \alpha$ line. The line broadening and redshift may arise from either Compton scattering in a cool plasma with small optical depth $(\tau \approx 5)$, or from Doppler and relativistic effects in the vicinity of a compact object. The spectrum below $2 \mathrm{keV}$, obtained with the TGS, shows evidence for a broad emission line feature at $0.74 \mathrm{keV}$, which may be an iron L-transition complex. However, we find that such an emission feature could be an artifact caused by an anomalously low interstellar absorption by neutral Oxygen. The continuum emission is extremely soft and is well described by an unsaturated Comptonized spectrum from a very cool plasma $(\mathrm{kT}=0.84 \mathrm{keV})$ with large scattering depth $(\tau=27)$. The continuum spectrum is strikingly similar to that of black hole candidate LMC X-3.
\end{abstract}

\section{INTRODUCTION AND OBSERVATIONS}

X-ray transients show dramatic outbursts in the X-ray band with typical recurrence times between 1 and 60 years and durations lasting several months. Their high luminosity $\left(10^{37}-10^{38} \mathrm{erg} \mathrm{s}^{-1}\right)$, optical counterparts, spectral characteristics and time variability during outburst are similar to the persistent bright $\mathrm{X}$-ray sources; close binary systems powered by accretion of matter onto a neutron star or black hole. White, Kaluzienski and Swank (1984) suggested that transients which show an "ultra-soft" (kT $\lesssim 3 \mathrm{keV}$ ) spectrum are good candidates for containing a black hole. The persistent $\mathrm{X}$-ray sources which show these ultra-soft spectra include the black hole candidates Cyg X-1 (in its high state) and LMC X-3 (White and Marshall 1984). The X-ray source 4U1543-47 was identified as an ultra-soft transient when it was detected in a high state in 1971 (Li, Sprott, and Clark 1976). Twelve years later another outburst from 4U1543-47 was discovered in 1983 August by the TENMA satellite (Tanaka 1983, Kitamoto et al. 1984). We report the results from high-resolution X-ray spectra taken with the European Space Agency's X-ray observatory EXOSAT (White and Peacock 1988). EXOSAT observed 4U1543-47 for 4 hours on 1983 August 28. The Gas Scintillation Proportional Counter (GSPC) detected the source to be bright with a counting rate $(2.0$ to $13.0 \mathrm{keV})$ at constant value of 335 counts $^{-1}$, or about one eigth the counting rate seen from Sco X-1. Spectra below $2 \mathrm{keV}$ were collected with the Transmission Grating Spectrometers (TGS) of the Low Energy imaging telecopes. We present the spectrum obtained with the LE1 spectrometer and thin Lexan filter, which has the best spectral resolution $(\Delta \mathrm{E} / \mathrm{E} \approx 10(\mathrm{E} / 1 \mathrm{keV}) \%)$ and photon statistics. 


\section{SPECTRAL RESULTS}

Various trial model spectra, folded through each detector response, were simultaneously fit to the GSPC spectrum and the negative and positive orders of the LE1 spectrum. For all combinations of many one and two component trial continuum models a broad emission line at $\sim 5.9 \mathrm{keV}$ in the GSPC was required. In the TGS an excess at $\sim 0.7 \mathrm{keV}$ was found. The continuum model with the least number of free parameters that gives an acceptable fit is a Comptonized spectrum as prescribed by Sunyaev and Titarchuk (1980), that includes absorption by neutral material with cosmic abundances (Morrison and McCammon 1983). The best fitting parameters (which also included two emission lines discussed below) give an electron temperature $\left(\mathrm{kT}_{e}\right)$ of $0.843 \pm 0.004 \mathrm{keV}$, an optical depth $(\tau)$, of $26.6 \pm 0.6$, and an equivalent hydrogen column density, $\mathrm{N}_{H}=(4.26 \pm 0.15) \times 10^{21} \mathrm{H}$ atoms $\mathrm{cm}^{-2}$ with a $\chi^{2}$ of 449 for 348 degrees of freedom (d.o.f.). Fig. 1 shows the model fit to both the GSPC and LE1 grating spectrum. For clarity only the LE1 negative orders are shown. The source luminosity in the band 0.4 to $13.0 \mathrm{keV}$, corrected for absorption, is $2.0 \times 10^{37} \mathrm{erg} \mathrm{s}^{-1}$, for an arbitrary distance of $1 \mathrm{kpc}$. The line in the GSPC was modelled using a Gausian profile and was centered at $5.93 \pm 0.24 \mathrm{keV}$ with a full width half maximum (FWHM) of $2.71 \pm 0.47 \mathrm{keV}$ and an equivalent width (EW) of $115 \mathrm{eV}$. The line profile is shown in Fig $1 \mathrm{~b}$, and is clearly a very broad feature. The structure in the residuals between 4 and $5 \mathrm{keV}$ arises from uncertainties in the calibration, but does not strongly influence the line parameters. The emission line in the TGS is at $0.742 \pm 0.027 \mathrm{keV}$ with $\mathrm{FWHM}=$ $0.204 \pm 0.068 \mathrm{keV}$, and $\mathrm{EW}=86 \mathrm{eV}$. The excess flux near $0.74 \mathrm{keV}$ can, however, also be interpreted as the result of an overestimation of the continuum absorption at this energy. The fit is sensitive to the depth of the oxygen K-edge at $0.53 \mathrm{keV}$ (Ride and Walker 1977), and an acceptable fit can be obtained by reducing the abundance of oxygen by $35 \%$, as compared to the solar abundance ( $\chi^{2}$ of 496 for 350 d.o.f.).

\section{DISCUSSION}

The broad feature around $0.7 \mathrm{keV}$ is either due to an emission line complex at 0.74 $\mathrm{keV}$, or due to depletion of the number of neutral oxygen atoms in the line of sight. In the former we find the line to be broad with a Gaussian FWHM of $0.2 \mathrm{keV}$. Since the energy resolution is only a factor of four better than this, the broad feature may be caused by the smearing together of an unresolved line complex, centered on $0.74 \mathrm{keV}$. The best candidates for such a complex would be the L-transitions of a wide spectrum of iron ions. Similar Fe L-shell complexes have been observed in other LMXB with the TGSs flown on EINSTEIN and EXOSAT (Kahn, Seward and Chlebowski 1984 (KSC), Brinkman et al. 1985, Vrtilek et al. 1986a,b). These lines are most likely formed by radiative recombination in a plasma which is photoionized by the strong continuum emission from the central source (KSC). An equally acceptable fit to the data is obtained without these lines, however, if oxygen is allowed to be underabundant by $65 \%$ from the cosmic value. It is notable that KSC also find in addition to the emission line features also an underabundance of oxygen for Sco X-1. It is possible that we are observing a combination of reduced abundance and an emission complex at $0.74 \mathrm{keV}$. Part of the observed absorption could be intrinsic to the system, in which case the apparent underabundance would reflect a real underabundance of oxygen within the accreting matter. Another possibility is that the observed absorption is interstellar and that oxygen is depleted due to grain formation (Fireman 1974, Ride and Walker 1977). When the grains are big enough they become opaque for soft X-rays and part of the grain is effectively shielded from the radiation. The average atom in the grain only absorbs $65 \%$ of the X-rays compared to a purely gaseous medium. This infers a typical grain size of $0.37 \mu$ in the direction of 4 U1543-47 (cf. Fireman 1974). 
LE1 Grating

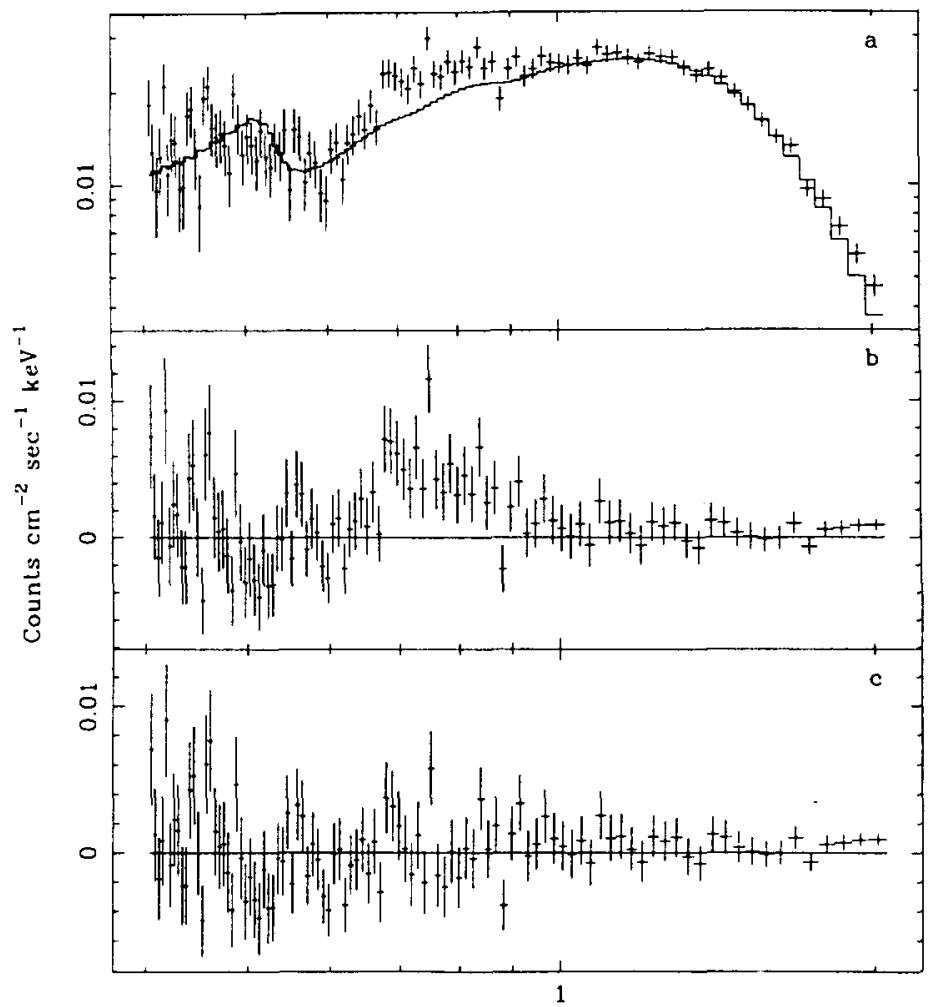

Channel Energy (keV)
Sunyaev \& Titarchuk

$\operatorname{GSPC}$

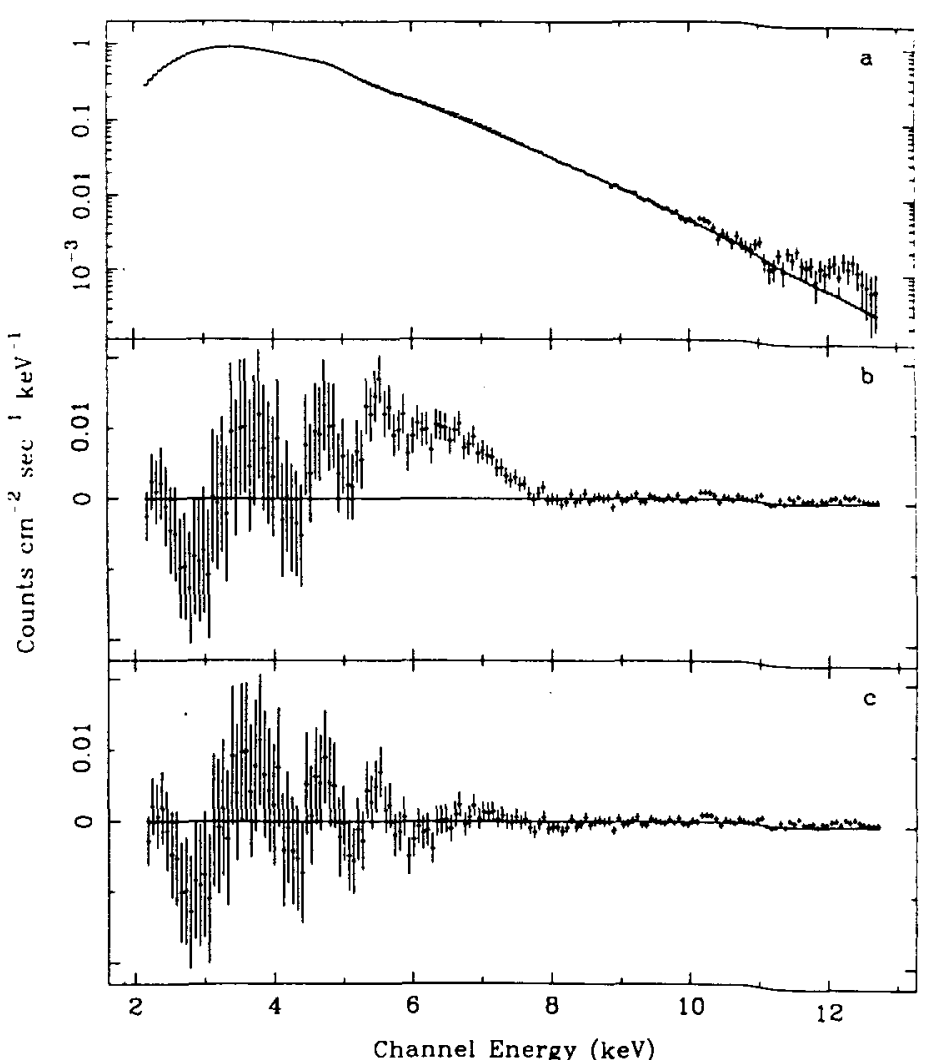

FIG. 1. The negative spectral orders of the LE1 grating spectrum and the GSPC spectrum of the soft X-ray transient 4U1543-47 near outburst maximum, fitted by an unsaturated comptonized spectrum (a). The (b) panels show the residuals, where the strengths of the emission feature at $0.74 \mathrm{keV}$ and of the iron line at $5.9 \mathrm{keV}$ was set to zero. Panels (c) shows the residuals for the fit with these emission features included. 
The broad emission feature seen in the GSPC centered on $5.9 \mathrm{keV}$ seems most likely associated with the iron $\mathrm{K}$-line complex between 6.4 and $6.7 \mathrm{keV}$. Broad iron $\mathrm{K}$-lines, typically with a FWHM of $1 \mathrm{keV}$ and line centroid at $6.4-6.7 \mathrm{keV}$, have been seen from several other LMXB (White et al. 1986, Hirano et al. 1987). An exception to this is the broad iron line discovered from the blackhole candidate Cyg X-1 by Barr, White and Page (1985) which is at $6.2 \mathrm{keV}$ with a FWHM of $1.2 \mathrm{keV}$. The broadening may arise from either Comptonization in an accretion disk corona (White et al. 1986, Hirano et al. 1987, Kallman and White 1989) or from the effects of rotation and general relativistic effects in the inner regions of an accretion disk, close to the compact object (Fabian et al. 1989). Comptonization in an optically thick accretion disk corona can explain the broadening and red shift, although Kallman and White (1989) have shown this requires a greater optical depth than that expected from a purely X-ray heated corona. If we consider only the recoil effect, which is certainly correct if the iron line is generated and broadened in a plasma with approximately the continuum temperature, we find that both the redshift and broadening are consistent with an optical depth of $\tau \approx 5$ (cf. Sunyaev and Titarchuk 1980).

The Comptonisation model (Titarchuk and Sunyaev 1980) found to give a good fit to the continuum spectrum of $4 U 1543-47$, is the same as that found for the spectrum of the blackhole candidate LMC X-3. The best fitting parameters are also very similar for 4U154347 with $\mathrm{kT}_{e}=0.84 \mathrm{keV}$ and $\tau=27$, which corresponds to a Comptonisation y parameter of 4.7 $\left(\mathrm{y}=4 k T_{e} \tau^{2} / m_{e} c^{2}\right)$ and for LMC X-3 kTe $\sim 1 \mathrm{keV}, \tau \sim 23$ and $\mathrm{y} \sim 4.1$ (Treves et al. 1988). This correspondence between the spectra of 4U1543-47 and LMC X-3 underlines the suggestion (White and Marshall 1984; White, Kaluzinski and Swank 1984) that ultrasoft transients should be considered good black holes candidates.

acknowledgements. We thank L. Chiappetti, R. Blissett, W. Craig and R. Rogers for contributions during an earlier phase of this work. SMK acknowledge support from a NASA Astrophysics Data Program. The EXOSAT Observatory team are thanked for their support. NEW thanks L. Stella and A. Fabian for discussions.

\section{REFERENCES}

Barr, P., White, N. E., and Page, C. G. 1985, M.N.R.A.S., 216, 65p.

Brinkman, A. C., et al. 1985, Space Sci. Rev., 40, 201.

Fabian, A.C., Rees, M.J., Stella, L., and White, N.E. 1989, M.N.R.A.S., in press.

Fireman, E. L. 1974, Ap. J., 187, 57.

Hirano, T., et al. 1987, Publ. Astron. Soc. Japan, 39, 619.

Kahn, S.M., Seward, F.D., and Chlebowski, T. 1984, Ap. J., 283, 286 (KSC).

Kallman, T.R., and White, N.E. $1989, A p$. J., in press.

Kitamoto, S., et al. 1984, . Publ. Astron. Soc. Japan, 36, 799.

Li, F. K., Sprott, G. F., and Clark, G. W. 1976, Ap. J., 203, 187.

Morrison, R., and McCammon, D. 1983, Ap. J., 270, 119.

Ride, S. K., and Walker, A. B. C. 1977, Astr. Ap., 61, 339.

Sunyaev, R. A., and Titarchuk, L. G. 1980, Astr. Ap., 86, 121.

Tanaka, Y. 1983, IAU Circ. No. 3854.

Treves, A. et al. 1988, Ap. J., 325, 119.

Vrtilek, S.D., et al. 1986a, Ap. J., 307, 698.

Vrtilek, S.D., et al. 1986b, Ap. J., 308, 644 .

White, N. E., and Marshall, F. E. 1984, Ap. J., 281, 354.

White, N. E., and Peacock, A. 1988, EXOSAT Preprint No. 75.

White, N. E., Kaluzienski, J. L., and Swank, J. H. 1984, in High Energy Transients in Astrophysics, ed. S. E. Woosley, AIP Conf. Proc. 115, p. 31.

White, N. E., et al. 1986, M.N.R.A.S., 218, 129. 